PROCEEDINGS OF THE

AMERICAN MATHEMATICAL SOCIETY

Volume 138, Number 10, October 2010, Pages 3541-3550

S 0002-9939(2010)10314-3

Article electronically published on June 4, 2010

\title{
TRACE-POSITIVE COMPLEX POLYNOMIALS IN THREE UNITARIES
}

\author{
STANISLAV POPOVYCH
}

(Communicated by Marius Junge)

\begin{abstract}
We consider the quadratic polynomials in three unitary generators, i.e. the elements of the group $*$-algebra of the free group with generators $u_{1}, u_{2}, u_{3}$ of the form $f=\sum_{j, k=1}^{3} \alpha_{j k} u_{j}^{*} u_{k}, \alpha_{j k} \in \mathbb{C}$. We prove that if $f$ is selfadjoint and $\operatorname{Tr}\left(f\left(U_{1}, U_{2}, U_{3}\right)\right) \geq 0$ for arbitrary unitary matrices $U_{1}, U_{2}, U_{3}$, then $f$ is a sum of hermitian squares. To prove this statement we reduce it to the question whether a certain Tarski sentence is true. Tarski's decidability theorem thus provides an algorithm to answer this question. We use an algorithm due to Lazard and Rouillier for computing the number of real roots of a parametric system of polynomial equations and inequalities implemented in Maple to check that the Tarski sentence is true.

As an application, we describe the set of parameters $a_{1}, a_{2}, a_{3}, a_{4}$ such that there are unitary operators $U_{1}, \ldots, U_{4}$ connected by the linear relation $a_{1} U_{1}+a_{2} U_{2}+a_{3} U_{3}+a_{4} U_{4}=0$.
\end{abstract}

\section{INTRODUCTION}

Let $R$ be a hyperfinite $\mathrm{II}_{1}$ factor and $\omega \in \beta(\mathbb{N}) \backslash \mathbb{N}$ be a free ultrafilter on $\mathbb{N}$. Connes' embedding conjecture states that every von Neumann $\mathrm{II}_{1}$ factor $\mathcal{M}$ with a separable predual and a fixed normal faithful tracial state can be embedded into the ultrapower $R^{\omega}$ in a trace-preserving way.

The following algebraic reformulation of the conjecture was obtained in [6].

Theorem. Let $\mathbb{F}$ denote the free group with a countable family of free generators $\left\{u_{1}, u_{2}, \ldots\right\}$. Denote by $\mathcal{F}$ its group $*$-algebra. Connes' embedding conjecture is true iff for any selfadjoint $f \in \mathcal{F}$ of the form

$$
f\left(u_{1}, \ldots, u_{n}\right)=\sum_{i, j=1}^{n} \alpha_{i j} u_{i}^{*} u_{j}, \quad \alpha_{i j} \in \mathbb{C}, i, j=1, \ldots, n,
$$

the condition

$$
\operatorname{Tr}\left(f\left(V_{1}, \ldots, V_{n}\right)\right) \geq 0
$$

for every $m \geq 1$ and every $n$-tuple of unitary $m \times m$ matrices $V_{1}, \ldots, V_{n}$ implies that

$$
\text { for every } \varepsilon>0, \varepsilon e+f=g+c,
$$

Received by the editors January 6, 2009 and, in revised form, November 11, 2009.

2000 Mathematics Subject Classification. Primary 46L10; Secondary 15A48.

Key words and phrases. Connes' Embedding Conjecture, $I I_{1}$-factor, trace, sum of hermitian squares, Tarski sentence, discriminant variety.

(C)2010 American Mathematical Society Reverts to public domain 28 years from publication 
where $e$ is the unit of $\mathcal{F}, c$ is a sum of commutators in $\mathcal{F}$ and $g$ is a sum of hermitian squares.

Here, a commutator means a ring commutator, i.e. an element of the form $g h-h g$ with $h, g \in \mathcal{F}$. A hermitian square in $\mathcal{F}$ is an element of the form $h^{*} h$ with $h \in \mathcal{F}$.

We will call the elements of the form $f=\sum_{i, j=1}^{n} \alpha_{i j} u_{i}^{*} u_{j}$ quadratic polynomials in unitary variables. If condition (1.1) holds, we will call $f$ trace-positive. An element $f$ such that $f\left(U_{1}, \ldots, U_{n}\right)$ is a positive operator for every unitary operator $U_{1}, \ldots, U_{n}$ will be called positive.

It was proved in 12 that every self-adjoint trace-positive quadratic polynomial with real coefficients is a sum of hermitian squares. In particular, every such polynomial is positive semidefinite. However, little is known about trace-positive self-adjoint quadratic polynomials with complex coefficients.

The aim of the present paper is to show that every trace-positive complex quadratic polynomial in three unitaries is a sum of hermitian squares. Firstly, we will reduce this statement to the validity of a Tarski sentence. It is well known that there exists an algorithm to check that a given Tarski sentence is true or false (see [14]). However, this algorithm is quite slow to be of practical help. Fortunately, there is an alternative approach based on computational methods of real algebraic geometry. In Section [2] we use the algorithm due to D. Lazard and F. Rouillier for counting the number of real roots of a multivariate system of polynomial equations and inequalities to prove the above result. It is based on the notion of a discriminant variety (see [9]) and has been implemented in Maple.

Note that a quadratic self-adjoint polynomial $f$ in 3 generators, by the change of variables $u=u_{1}^{*} u_{2}, v=u_{1}^{*} u_{3}$, can be presented in the form $f(u, v)=a_{0}+a u+$ $\bar{a} u^{*}+b v+\bar{b} v^{*}+c u^{*} v+\bar{c} v^{*} u$ with $a_{0} \in \mathbb{R}, a, b, c \in \mathbb{C}$. Thus, $f$ is trace-positive if and only if

$$
a_{0}+2 \operatorname{Re}\left(a \operatorname{tr}(U)+b \operatorname{tr}(V)+c \operatorname{tr}\left(U^{*} V\right)\right) \geq 0
$$

for all unitary matrices $U, V \in M_{m}(\mathbb{C})$ with arbitrary $m \geq 1$. Here, $\operatorname{tr}(\cdot)=\frac{1}{m} \operatorname{Tr}(\cdot)$ denotes the normalized trace. Thus, a description of the trace-positive of the trace positive polynomials in 3 variables amounts to a description of the closure in $\mathbb{C}^{3}$ of the set

$$
\begin{aligned}
T=\left\{\left(\operatorname{tr}(U), \operatorname{tr}(V), \operatorname{tr}\left(U^{*} V\right)\right) \mid\right. & m \geq 1, U, V \in \mathcal{U}(m)\} \\
= & \{(\operatorname{tr}(U), \operatorname{tr}(V), \operatorname{tr}(U V)) \mid m \geq 1, U, V \in \mathcal{U}(m)\},
\end{aligned}
$$

where $\mathcal{U}(m)$ denotes the group of the unitary $m \times m$ matrices.

Note that the lists of the possible eigenvalues of $U, V$ and $U V$ can be completely described by a generalization of Horn's inequalities (see [1, 5]). However, only a simple connection between the normalized traces of two unitaries and their product has been known until recently. It is the inequality

$$
\sqrt{1-|\operatorname{tr}(U V)|^{2}} \leq \sqrt{1-|\operatorname{tr}(U)|^{2}}+\sqrt{1-|\operatorname{tr}(V)|^{2}}
$$

established in [15].

Let $\Theta_{n}$ denote the set of all positive semidefinite matrices with 1's on the main diagonal. Let $\mathcal{F}_{n}$ denote the closure of the set of matrices

$$
\left\{\left(\operatorname{tr}\left(U_{k}^{*} U_{j}\right)\right)_{k j} \mid m \in \mathbb{N}, U_{1}, \ldots, U_{n} \in \mathcal{U}(m)\right\}
$$


where $\mathcal{U}(m)$ is the set of the unitary $m \times m$ matrices. Clearly, $\mathcal{F}_{n} \subseteq \Theta_{n}$. It can be easily inferred from the results of [4 that the set $T$ is closed, and thus the map

$$
(a, b, c) \mapsto\left(\begin{array}{ccc}
1 & a & b \\
\bar{a} & 1 & c \\
\bar{b} & \bar{c} & 1
\end{array}\right)
$$

is one-to-one from $T$ onto $\mathcal{F}_{3}$. A complete description of $\mathcal{F}_{3}$ was obtained in [4]. However, no description of the set $\mathcal{F}_{n}$ for $n>3$ is known at present. Let $\mathcal{F}_{n}^{c}$ denote the closure of the set of matrices $\left(\operatorname{tr}\left(U_{k}^{*} U_{m}\right)\right)_{k, m=1}^{n}$, where unitary matrices $U_{1}, \ldots, U_{n} \in \mathcal{U}(d)$ (of arbitrary size $d \geq 1$ ) commute:

$$
U_{k} U_{m}=U_{m} U_{k} \text { for all } k, m=1, \ldots, n \text {. }
$$

The set $\mathcal{F}_{n}^{c}$ can be alternatively described as the convex hull of the rank one matrices from $\Theta_{n}$ (see [4]).

The main result of the paper is the following:

Theorem 1.1. For a self-adjoint quadratic polynomial

$$
f\left(u_{1}, u_{2}, u_{3}\right)=a_{0}+a u_{1}^{*} u_{2}+\bar{a} u_{2}^{*} u_{1}+b u_{1}^{*} u_{3}+\bar{b} u_{3}^{*} u_{1}+c u_{2}^{*} u_{3}+\bar{c} u_{3}^{*} u_{2}
$$

the following statements are equivalent:

(1) $f\left(z_{1}, z_{2}, z_{3}\right) \geq 0$ for $z_{1}, z_{2}, z_{3} \in \mathbb{C}$ and $\left|z_{1}\right|=\left|z_{2}\right|=\left|z_{3}\right|=1$.

(2) $f$ is trace-positive.

(3) $f$ is positive.

(4) $f$ is a sum of hermitian squares.

As a byproduct of Lemma 2.1 we also show the equality $\Theta_{3}=\mathcal{F}_{3}=\mathcal{F}_{3}^{c}$ proved earlier in [4 by different methods.

Remark 1.2. It was also shown in 4 that $\mathcal{F}_{6} \neq \mathcal{F}_{6}^{c}$. Thus, for self-adjoint quadratic polynomials $f$ in 6 unitary variables the condition that $f\left(z_{1}, \ldots, z_{4}\right) \geq 0$ for every $z_{1}, \ldots, z_{6} \in \mathbb{C}$ with $\left|z_{1}\right|=\ldots=\left|z_{6}\right|=1$ does not imply that $f$ is trace-positive. It is still unknown, however, whether $\mathcal{F}_{4}=\mathcal{F}_{4}^{c}$.

A polynomial $f$ of the form (1.3) will be called $\Theta_{3}$-positive if $a_{0}+2 \operatorname{Re}(a \alpha+b \beta+$ $c \gamma) \geq 0$ for every matrix

$$
A=\left(\begin{array}{ccc}
1 & \alpha & \beta \\
\bar{\alpha} & 1 & \gamma \\
\bar{\beta} & \bar{\gamma} & 1
\end{array}\right) \in \Theta_{3} .
$$

Clearly, every $\Theta_{3}$-positive polynomial is trace-positive. Put $Q(a, b, c, A)=\operatorname{Re}(a \alpha+$ $b \beta+c \gamma)$ and $P(a, b, c, \phi, \psi)=\operatorname{Re}\left(a e^{i \phi}+b e^{i \psi}+c e^{i(\psi-\phi)}\right)$.

Since for every $\Theta_{3}$-positive $f$ we have

$$
a_{0}+2 \operatorname{Re}\left(a \operatorname{tr}\left(V_{1}\right)+b \operatorname{tr}\left(V_{2}\right)+c \operatorname{tr}\left(V_{1}^{*} V_{2}\right)\right) \geq 0
$$

with $V_{1}=e^{i \phi}, V_{2}=e^{i \psi}$, we get the following inequality:

$$
\min _{A \in \Theta_{3}} Q(a, b, c, A) \leq \min _{\phi, \psi \in[0,2 \pi)} P(a, b, c, \phi, \psi) .
$$

Meanwhile, if $a_{0} \geq x_{1}+x_{2}+x_{3}$ for some $x_{1}, x_{2}, x_{3}$ such that the matrix

$$
B=\left(\begin{array}{ccc}
x_{1} & a & b \\
\bar{a} & x_{2} & c \\
\bar{b} & \bar{c} & x_{3}
\end{array}\right) \geq 0,
$$


then the Schur product $B \circ A$ is positive semidefinite for every $A \in \Theta_{3}$ and, consequently,

$$
x_{1}+x_{2}+x_{3}+2 \operatorname{Re}(a \alpha+b \beta+c \gamma)=(1,1,1)(B \circ A)(1,1,1)^{T} \geq 0 .
$$

Hence,

$$
\begin{aligned}
\min \left\{x_{1}+x_{2}+x_{3} \mid\left(\begin{array}{ccc}
x_{1} & a & b \\
\bar{a} & x_{2} & c \\
\bar{b} & \bar{c} & x_{3}
\end{array}\right) \geq 0\right\} & \geq \max _{A \in \Theta_{3}}(-2 Q(a, b, c, A)) \\
& \geq \max _{\phi, \psi \in[0,2 \pi)}(-2 P(a, b, c, \phi, \psi)) .
\end{aligned}
$$

\section{Proofs}

Lemma 2.1. For every $a, b, c \in \mathbb{C}$ the following formula holds:

$$
\min \left\{x_{1}+x_{2}+x_{3} \mid\left(\begin{array}{ccc}
x_{1} & a & b \\
\bar{a} & x_{2} & c \\
\bar{b} & \bar{c} & x_{3}
\end{array}\right) \geq 0\right\}=\max _{\phi, \psi \in[0,2 \pi)}(-2 P(a, b, c, \phi, \psi)) .
$$

Remark 2.2. The analog of formula (2.1) for the $4 \times 4$ matrices does not hold (see Remark 2.5).

Proof. By inequalities (1.4)-(1.5), the above equality holds if and only if there exists $x_{1}, x_{2}, x_{3}, \phi, \psi$ such that

$$
\begin{gathered}
x_{1}+x_{2}+x_{3}=-2 \operatorname{Re}\left(a e^{i \phi}+b e^{i \psi}+c e^{i(\psi-\phi)}\right), \\
\left(\begin{array}{ccc}
x_{1} & a & b \\
\bar{a} & x_{2} & c \\
\bar{b} & \bar{c} & x_{3}
\end{array}\right) \geq 0 .
\end{gathered}
$$

Clearly, it is enough to prove the above formula for $a \geq 0$ and $b \geq 0$ and $c=c_{r}+i c_{i}$ with $c_{r}, c_{i} \in \mathbb{R}$. This restriction will be assumed in the sequel.

If $x_{1}, x_{2}, x_{3}, \phi, \psi$ satisfy (2.2)-(2.3), then

$$
A=\left(\begin{array}{ccc}
x_{1} & a & b \\
\bar{a} & x_{2} & c \\
\bar{b} & \bar{c} & x_{3}
\end{array}\right) \geq 0
$$

Hence, the matrix $X A X^{*} \geq 0$ for every matrix $X$. With $X=\operatorname{diag}\left(1, e^{i \phi}, e^{i \psi}\right)$, we have

$$
B=X A X^{*}=\left(\begin{array}{ccc}
x_{1} & a e^{i \phi} & b e^{i \psi} \\
\bar{a} e^{-i \phi} & x_{2} & c e^{i(\psi-\phi)} \\
\bar{b} e^{-i \psi} & \bar{c} e^{-i(\psi-\phi)} & x_{3}
\end{array}\right) \geq 0 .
$$

It is easy to check that equation (2.2) is equivalent to $(1,1,1) B(1,1,1)^{T}=0$. The positivity of $B$ implies that $B(1,1,1)^{T}=0$. Hence,

$$
\begin{gathered}
x_{1}=-a e^{i \phi}-b e^{i \psi}, \\
x_{2}=-\bar{a} e^{-i \phi}-c e^{i(\psi-\phi)}, \\
x_{3}=-\bar{b} e^{-i \psi}-\bar{c} e^{-i(\psi-\phi)} .
\end{gathered}
$$


Write $e^{i \phi}=u_{1}+i v_{1}$ and $e^{i \psi}=u_{2}+i v_{2}$. The conditions

$$
\begin{gathered}
u_{1}^{2}+v_{1}^{2}=1, u_{2}^{2}+v_{2}^{2}=1, \\
\operatorname{Im}\left(x_{1}\right)=\operatorname{Im}\left(x_{2}\right)=\operatorname{Im}\left(x_{3}\right)=0
\end{gathered}
$$

are equivalent to the following formulas:

$$
\begin{gathered}
u_{1}=-\frac{c_{r} x b\left(\left(b^{2}\left(a^{2}-|c|^{2}\right)+a^{2}|c|^{2}\right) x^{2}-2 x a^{3} c_{i}+a^{2} c_{i}^{2}\right)}{2 b^{2} a^{2}|c|^{2} x^{3}-\left(b^{2} a^{2}+\left(b^{2}+a^{2}\right)|c|^{2}\right) a c_{i} x^{2}+a^{3} c_{i}^{3}}, \\
v_{1}=\frac{-b x}{a}, \\
u_{2}=\frac{1}{2} \frac{2 b^{2} a c_{i} x^{3}+\left(-a^{2} b^{2}+a^{2}\left(c_{r}^{2}-c_{i}^{2}\right)-b^{2}|c|^{2}\right) x^{2}+a^{2} c_{i}^{2}}{x a c_{r}\left(x b^{2}-a c_{i}\right)}, \\
v_{2}=x,
\end{gathered}
$$

where $x$ is any real root of the polynomial

$$
\begin{gathered}
q(x)=4 b^{4} a^{2}|c|^{2} x^{6}-4 a b^{2} c_{i}\left(b^{2}|c|^{2}+a^{2}|c|^{2}+b^{2} a^{2}\right) x^{5} \\
+\left(4 b^{2} a^{3}\left(c^{2}+c_{r}^{2}\right) c_{i}+2 b^{4} a^{2}|c|^{2}+\left(a^{2}+b^{2}\right)^{2}|c|^{4}\right. \\
\left.-b^{2} a^{2}\left(4|c|^{2} c_{r}^{2}+2 a^{2}|c|^{2}-4 a^{2} c_{r}^{2}-4 c_{r}^{2} b^{2}+a^{2} b^{2}\right)\right) x^{4} \\
+4 b^{2} a^{3} c_{i}\left(c_{i}^{2}+2 c_{r}^{2}\right) x^{3} \\
-2 a^{2}\left(|c|^{2}-c_{r}^{2}\right)\left(b^{2}|c|^{2}+a^{2} c^{2}+b^{2} a^{2}\right) x^{2}+a^{4} c_{i}^{4} .
\end{gathered}
$$

The substitution of the above expressions for $u_{1}, v_{1}, u_{2}, v_{2}$ into the formulas for $x_{1}, x_{2}, x_{3}$ and then into the matrix $B$ gives us an expression for the coefficients of $B$ as rational functions depending on $a, b, c_{r}, c_{i}, x$. Thus,

$$
\begin{gathered}
b_{11}=\frac{p_{1}\left(a, b, c_{r}, c_{i}, x\right)}{q_{1}\left(a, b, c_{r}, c_{i}, x\right)}, \\
\operatorname{det}\left(\begin{array}{cc}
b_{11} & b_{12} \\
b_{21} & b_{22}
\end{array}\right)=\frac{p_{2}\left(a, b, c_{r}, c_{i}, x\right)}{q_{2}\left(a, b, c_{r}, c_{i}, x\right)}, \\
\operatorname{det} B=0
\end{gathered}
$$

where $p_{1}, p_{2}, q_{1}, q_{2}$ are some polynomials in $a, b, c_{r}, c_{i}, x$ which can be easily computed explicitly. Thus, formulas (2.2)-(2.3) are valid if the following Tarski sentence is true:

$$
\begin{gathered}
\forall a \forall b \forall c_{r} \forall c_{i} \exists x q(x)=0 \& p_{1}\left(a, b, c_{r}, c_{i}, x\right) q_{1}\left(a, b, c_{r}, c_{i}, x\right)>0 \\
\& p_{2}\left(a, b, c_{r}, c_{i}, x\right) q_{2}\left(a, b, c_{r}, c_{i}, x\right)>0 .
\end{gathered}
$$

We need to prove that $q(x)$ has at least one real root for arbitrary parameters $a, b, c$. 
However, there exists an algorithm (see 14]) to check the validity of any Tarski sentence, but it is quite slow in practice. We find it more efficient to use an algorithm for counting the number of real roots of a multivariate system of polynomial equations and inequalities based on the notion of a discriminant variety (see [9]).

For a basic semi-algebraic set

$$
\mathcal{S}=\left\{x \in \mathbb{R}^{n} \mid p_{1}(x)=0, \ldots, p_{s}(x)=0, f_{1}(x)>0, \ldots, f_{l}(x)>0\right\},
$$

and a basic constructable set

$$
\mathcal{C}=\left\{x \in \mathbb{R}^{n} \mid p_{1}(x)=0, \ldots, p_{s}(x)=0, f_{1}(x) \neq 0, \ldots, f_{l}(x) \neq 0\right\}
$$

where $p_{j}, f_{t}$ are polynomials with rational coefficients, the notion of discriminant variety has been introduced in 9 .

The indeterminates $x=\left(U_{1}, \ldots, U_{d}, X_{d+1}, \ldots X_{n}\right)$ are partitioned into two sets: the parameters $\left(U_{1}, \ldots, U_{d}\right)$ and the unknowns $\left(X_{d+1}, \ldots, X_{n}\right)$. Let $\Pi_{U}: \mathbb{C}^{n} \rightarrow \mathbb{C}^{d}$,

$$
\Pi_{U}\left(U_{1}, \ldots, U_{d}, X_{d+1}, \ldots X_{n}\right)=\left(U_{1}, \ldots, U_{d}\right)
$$

be the canonical projection on the parameter space. For a given ideal $I$ in the ring of (commutative) polynomials, we denote by $\mathbf{V}(I)$ the associated algebraic variety. Let $\overline{\Pi_{U}(\mathcal{C})}$ be the closure of $\Pi_{U}(\mathcal{C})$ in the topology of $\mathbb{C}^{n}$ or Zariski closure (which are the same [11]).

Definition 2.3 (Lazard, Rouillier). An algebraic variety $W$ is a discriminant $v a$ riety of $\mathcal{C}$ with respect to $\Pi_{U}$ if

(1) $W \subseteq \overline{\Pi_{U}(\mathcal{C})}$.

(2) $W=\overline{\Pi_{U}(\mathcal{C})}$ if and only if $\Pi_{U}^{-1}(u) \cap \mathcal{C}$ is infinite for almost all $u \in \overline{\Pi_{U}(\mathcal{C})}$.

(3) The connected components $\mathcal{U}_{1}, \ldots, \mathcal{U}_{k}$ of $\overline{\Pi_{U}(\mathcal{C})} \backslash W$ are analytic submanifolds (i.e., nonsingular) of dimension $\delta$.

(4) $\left(\Pi_{U}^{-1}\left(\mathcal{U}_{i}\right) \cap \mathcal{C}, \Pi_{U}\right)$ is an analytic covering for $\mathcal{U}_{i}$ for all $i$.

There is an algorithm for computing the minimal discriminant variety described in [9]. It has been implemented as Maple package RootFinding [Parametric]. In particular, for the constructable set

$$
\left\{q(x)=0, p_{1}\left(a, b, c_{r}, c_{i}, x\right) q_{1}\left(a, b, c_{r}, c_{i}, x\right)>0, p_{2}\left(a, b, c_{r}, c_{i}, x\right) q_{2}\left(a, b, c_{r}, c_{i}, x\right)>0\right\},
$$

the minimal discriminant variety is the union

$$
\begin{gathered}
\mathbf{V}(a) \cup \mathbf{V}(b) \cup \mathbf{V}\left(c_{i}\right) \cup \mathbf{V}\left(c_{r}\right) \cup \mathbf{V}(a-b) \cup \mathbf{V}(a+b) \\
\cup \mathbf{V}\left(c_{r}^{2}+c_{i}^{2}\right) \cup \mathbf{V}\left(b^{2}-c_{r}^{2}-c_{i}^{2}\right) \cup \mathbf{V}\left(a^{2}-c_{r}^{2}-c_{i}^{2}\right) \cup \mathbf{V}(f),
\end{gathered}
$$


where

$$
\begin{gathered}
f=\left(6 a^{10} b^{2}+20 a^{6} b^{6}+b^{12}+6 a^{2} b^{10}+a^{12}+15 a^{8} b^{4}+15 a^{4} b^{8}\right)|c|^{12} \\
+\left(-12 a^{10} b^{2} c_{r}^{2}+6 a^{12} b^{2}-48 a^{4} b^{8} c_{r}^{2}-12 a^{2} b^{10} c_{r}^{2}-48 a^{8} c_{r}^{2} b^{4}\right. \\
\left.-102 a^{6} b^{8}-24 a^{10} b^{4}+6 a^{2} b^{12}-102 a^{8} b^{6}-24 a^{4} b^{10}-72 a^{6} c_{r}^{2} b^{6}\right)|c|^{10} \\
+\left(495 a^{8} b^{8}+15 a^{4} b^{12}+60 a^{6} c_{r}^{2} b^{8}-102 a^{6} b^{10}+15 a^{12} b^{4}\right. \\
+48 a^{4} b^{8} c_{r}^{4}-12 a^{12} c_{r}^{2} b^{2}-12 a^{2} c_{r}^{2} b^{12}+96 a^{6} c_{r}^{4} b^{6}+48 a^{8} c_{r}^{4} b^{4}+60 a^{8} c_{r}^{2} b^{6} \\
\left.-102 a^{10} b^{6}+216 a^{4} b^{10} c_{r}^{2}+216 a^{10} b^{4} c_{r}^{2}\right)|c|^{8} \\
+\left(-174 a^{10} b^{4} c_{r}^{4}-48 a^{12} b^{4} c_{r}^{2}+6 a^{6} c_{r}^{4} b^{8}-102 a^{10} b^{8}-174 a^{4} b^{10} c_{r}^{4}\right. \\
-432 a^{8} c_{r}^{2} b^{8}-64 a^{6} c_{r}^{6} b^{6}+20 a^{12} b^{6}-48 a^{4} b^{12} c_{r}^{2}-102 a^{8} b^{10}+20 a^{6} b^{12} \\
\left.+6 a^{8} c_{r}^{4} b^{6}+60 a^{10} b^{6} c_{r}^{2}+60 a^{6} c_{r}^{2} b^{10}\right)|c|^{6} \\
+\left(-72 a^{6} c_{r}^{2} b^{12}+24 a^{6} c_{r}^{6} b^{8}+15 a^{12} b^{8}+60 a^{8} c_{r}^{2} b^{10}\right. \\
+24 a^{8} c_{r}^{6} b^{6}+6 a^{10} b^{6} c_{r}^{4}-24 a^{10} b^{10}-90 a^{8} c_{r}^{4} b^{8} \\
\left.+60 a^{10} b^{8} c_{r}^{2}+15 a^{8} b^{12}+48 a^{12} b^{4} c_{r}^{4}-72 a^{12} b^{6} c_{r}^{2}+48 a^{4} b^{12} c_{r}^{4}+6 a^{6} c_{r}^{4} b^{10}\right)|c|^{4} \\
+\left(48 a^{8} c_{r}^{6} b^{8}+6 a^{8} c_{r}^{4} b^{10}+96 a^{12} c_{r}^{4} b^{6}+6 a^{10} b^{8} c_{r}^{4}-48 a^{12} c_{r}^{2} b^{8}-48 a^{8} c_{r}^{2} b^{12}\right. \\
\left.+24 a^{6} c_{r}^{6} b^{10}+24 a^{10} b^{6} c_{r}^{6}+216 a^{10} b^{10} c_{r}^{2}+96 a^{6} c_{r}^{4} b^{12}+6 a^{10} b^{12}+6 a^{12} b^{10}\right)|c|^{2} \\
+24 a^{8} c_{r}^{6} b^{10}-64 a^{6} c_{r}^{6} b^{12}+48 a^{12} c_{r}^{4} b^{8}-12 a^{12} c_{r}^{2} b^{10} \\
-64 a^{12} c_{r}^{6} b^{6}-174 a^{10} b^{10} c_{r}^{4}+24 a^{10} b^{8} c_{r}^{6}-12 a^{10} b^{12} c_{r}^{2} \\
+48 a^{8} c_{r}^{4} b^{12}+a^{12} b^{12}-27 a^{8} c_{r}^{8} b^{8} . \\
+
\end{gathered}
$$

The algorithm also provides us with an open cylindrical algebraic decomposition (see [3]) of the set $T=\left(\overline{\Pi_{U}(\mathcal{C})} \backslash W\right) \cap \mathbb{R}^{4}$ compatible with the set of polynomials $F$ defining $W$. This gives a representation of $T$ as a disjoint finite union of cells (semialgebraic sets isomorphic to $\mathbb{R}^{4}$ ). Each cell is contained in a connected component of $T$, and each connected component of $T$ has the same closure as the union of the cells it contains. Moreover, each polynomial in $F$ has a constant sign on each cell of the decomposition. The number of real solutions is constant for parameters belonging to the same cell.

In our case there are 2688 cells, the number of real solutions ranging between 1 and 3. In particular, this calculation proves that system (2.2)-(2.3) has a solution $x_{1}, x_{2}, x_{3}, \phi, \psi$ for every vector of parameters in $T$. Hence, equation (2.1) holds for every $(a, b, c) \in T$.

Since both the left-hand side and the right-hand side of (2.1) are, clearly, continuous functions in $a, b, c_{r}, c_{i}$, it is therefore sufficient to check formula (2.1) for $\left(a, b, c_{r}, c_{i}\right)$ belonging to some Zariski open subset. In particular, we can take the subset $T$. This proves that (2.1) holds for all complex $a, b, c$.

Proof of Theorem 1.1. If $f\left(z_{1}, z_{2}, z_{3}\right) \geq 0$ for complex $z_{1}, z_{2}, z_{3}$ of modulus one, then $a_{0}+2 \operatorname{Re}\left(a e^{i \phi}+b e^{i \psi}+c e^{i(\psi-\phi)}\right) \geq 0$ for all $\phi, \psi \in \mathbb{R}$. By Lemma 2.1, there are $x_{1}, x_{2}, x_{3} \in \mathbb{R}$ such that $a_{0} \geq x_{1}+x_{2}+x_{3}$ and the matrix

$$
\left(\begin{array}{ccc}
x_{1} & a & b \\
\bar{a} & x_{2} & c \\
\bar{b} & \bar{c} & x_{3}
\end{array}\right)
$$


is positive semidefinite. From [12, Lemma 4] it follows that $f$ is a sum of hermitian squares. This proves the implication $(1) \Rightarrow(4)$. The rest of the equivalences follow easily.

Corollary 2.4. $\Theta_{3}=\mathcal{F}_{3}=\mathcal{F}_{3}^{c}$.

Proof. Clearly $\mathcal{F}_{3}^{c} \subseteq \mathcal{F}_{3} \subseteq \Theta_{3}$. The sets $\Theta_{3}$ and $\mathcal{F}_{3}^{c}$ are closed convex sets in $\mathbb{C}^{3}$. If we assume that $\mathcal{F}_{3}^{c} \varsubsetneqq \Theta_{3}$, then we can find a linear functional $F: \mathbb{C}^{3} \rightarrow \mathbb{R}$ and a real number $t$ such that $F(v) \geq t$ for $v \in \mathcal{F}_{3}^{c}$ but $F(w)<t$ for some $w \in \Theta_{3}$. Clearly, the functional $F$ can be represented as $F(\alpha, \beta, \gamma)=\operatorname{Re}(\alpha a+\beta b+\gamma c)$ for some $a, b, c \in \mathbb{C}$. From (1.5) and Lemma 2.1, we have the equality

$$
\max _{A \in \Theta_{3}}(-Q(a, b, c, A))=\max _{\phi, \psi \in[0,2 \pi)}(-P(a, b, c, \phi, \psi)) \text {. }
$$

This gives a contradiction since the left-hand side of this equality is greater than $-t$ and the right-hand side is less than or equal to $-t$.

Remark 2.5. If the natural analog of Lemma 2.1 for the $4 \times 4$ matrices were valid, then, as in the proof above, we would have $\Theta_{4}=\mathcal{F}_{4}^{c}$. However, it was shown in [4] that $\Theta_{4} \neq \mathcal{F}_{4}^{c}$. Thus, Lemma 2.1 cannot be generalized for the matrices of bigger size.

\section{Application. Linear combinations of Four Unitaries}

For $\beta_{1}, \ldots, \beta_{4} \in \mathbb{C}$, consider the $*$-algebra given by the generators and relations

$$
\mathcal{U}_{\beta_{1}, \beta_{2}, \beta_{3}, \beta_{4}}=\mathbb{C}\left\langle u_{1}, u_{2}, u_{3}, u_{4} \mid \beta_{1} u_{1}+\beta_{2} u_{2}+\beta_{3} u_{3}+\beta_{4} u_{4}=0\right\rangle .
$$

As an application of Lemma 2.1, we get the following theorem.

Theorem 3.1. Let $\beta_{1}, \ldots, \beta_{4} \in \mathbb{C}$. The $*$-algebra $\mathcal{U}_{\beta_{1}, \beta_{2}, \beta_{3}, \beta_{4}}$ has a representation on a Hilbert space $H$ if and only if it has a one-dimensional representation.

Proof. Let $\pi: \mathcal{U}_{\beta_{1}, \beta_{2}, \beta_{3}, \beta_{4}} \rightarrow B(H)$ be a representation. Then the unitary operators $U_{k}=\pi\left(u_{k}\right)(k=1,2,3,4)$, acting in a Hilbert space $H$, satisfy the linear relation

$$
\beta_{1} U_{1}+\cdots+\beta_{4} U_{4}=0 .
$$

To simplify notation, we will rewrite this relation in the form

$$
Z=\alpha_{1} U+\alpha_{2} V+\alpha_{3} W
$$

with $U, V, W, Z$ unitary operators. Without loss of generality, we can assume that $\alpha_{1}, \alpha_{2}, \alpha_{3}$ are real. We need to show that there are $\phi_{1}, \ldots, \phi_{4} \in \mathbb{R}$ such that

$$
e^{i \phi_{4}}=\alpha_{1} e^{i \phi_{1}}+\alpha_{2} e^{i \phi_{2}}+\alpha_{3} e^{i \phi_{3}} .
$$

Let $u, v, w, z$ be the unit vectors which are the first columns of the operators $U$, $V, W, Z$ which are given by (possibly infinite) matrices in some orthonormal basis. Then $z=\alpha u+\beta v+\gamma w$. Thus,

$$
\left\langle\alpha_{1} u+\alpha_{2} v+\alpha_{3} w, \alpha_{1} u+\alpha_{2} v+\alpha_{3} w\right\rangle=1 .
$$

Hence,

$$
\operatorname{Re}\left(\alpha_{1} \alpha_{2}\langle u, v\rangle+\alpha_{1} \alpha_{3}\langle u, w\rangle+\alpha_{2} \alpha_{3}\langle v, w\rangle\right)=\frac{1}{2}\left(1-\alpha_{1}^{2}-\alpha_{2}^{2}-\alpha_{3}^{2}\right) .
$$

Consider the set $X_{\alpha_{1}, \alpha_{2}, \alpha_{3}}$ consisting of the real numbers

$$
2 \operatorname{Re}\left(\alpha_{1} \alpha_{2}\left\langle u_{1}, u_{2}\right\rangle+\alpha_{1} \alpha_{3}\left\langle u_{1}, u_{3}\right\rangle+\alpha_{2} \alpha_{3}\left\langle u_{2}, u_{3}\right\rangle\right),
$$


where $u_{1}, u_{2}, u_{3}$ runs over all triples of unit vectors in a separable Hilbert space. Since $(H, \operatorname{Re}\langle\cdot, \cdot\rangle)$ defines a real Hilbert space, the set $X_{\alpha_{1}, \alpha_{2}, \alpha_{3}}$ is the same as the set

$$
\left\{2\left(\alpha_{1} \alpha_{2}\left\langle u_{1}, u_{2}\right\rangle+\alpha_{1} \alpha_{3}\left\langle u_{1}, u_{3}\right\rangle+\alpha_{2} \alpha_{3}\left\langle u_{2}, u_{3}\right\rangle\right) \mid\left(u_{1}, u_{3}, u_{3}\right) \in \mathcal{U}_{3}\right\},
$$

where $\mathcal{U}_{3}$ consists of triples of unit vectors in a separable real Hilbert space.

The set consisting of the matrices

$$
\left(\begin{array}{ccc}
1 & \left\langle u_{1}, u_{2}\right\rangle & \left\langle u_{1}, u_{3}\right\rangle \\
\left\langle u_{1}, u_{2}\right\rangle & 1 & \left\langle u_{2}, u_{3}\right\rangle \\
\left\langle u_{1}, u_{3}\right\rangle & \left\langle u_{2}, u_{3}\right\rangle & 1
\end{array}\right)
$$

coincides with the real positive semidefinite matrices with 1's on the main diagonal, which is a convex set. Thus, $X_{\alpha_{1}, \alpha_{2}, \alpha_{3}}$ must be a closed convex bounded subset of $\mathbb{R}$. Let $a=\min X, b=\max X$, i.e. $X_{\alpha_{1}, \alpha_{2}, \alpha_{3}}=[a, b]$.

It was proved in [12] that for any set of coefficients $\left(a_{i j}\right)_{i<j} \in \mathbb{R}^{n(n-1) / 2}$ one has

$$
\begin{aligned}
& \min \left\{x_{1}+\cdots+x_{n} \mid\left(\begin{array}{cccc}
x_{1} & a_{12} & \ldots & a_{1 n} \\
a_{12} & x_{2} & \ldots & a_{2 n} \\
\vdots & \vdots & \ddots & \vdots \\
a_{1 n} & a_{2 n} & \ldots & x_{n}
\end{array}\right) \geq 0\right\} \\
= & -\min \left\{2 \sum_{\substack{i, j=1 \\
i<j}}^{n} a_{i j} q_{i j} \mid\left(\begin{array}{cccc}
1 & q_{12} & \ldots & q_{1 n} \\
q_{12} & 1 & \ldots & q_{2 n} \\
\vdots & \vdots & \ddots & \vdots \\
q_{1 n} & q_{2 n} & \ldots & 1
\end{array}\right) \geq 0\right\} .
\end{aligned}
$$

In particular, we have that

$$
\begin{aligned}
2 \max & \left\{\alpha_{1} \alpha_{2}\left\langle u_{1}, u_{2}\right\rangle+\alpha_{1} \alpha_{3}\left\langle u_{1}, u_{3}\right\rangle+\alpha_{2} \alpha_{3}\left\langle u_{2}, u_{3}\right\rangle \mid\left(u_{1}, u_{3}, u_{3}\right) \in \mathcal{U}_{3}\right\} \\
& =\min \left\{y_{1}+y_{2}+y_{3} \mid\left(\begin{array}{ccc}
1 & \alpha_{1} \alpha_{2} & -\alpha_{1} \alpha_{3} \\
-\alpha_{1} \alpha_{2} & 1 & -\alpha_{2} \alpha_{3} \\
-\alpha_{1} \alpha_{3} & -\alpha_{2} \alpha_{3} & 1
\end{array}\right) \geq 0\right\} \\
& =-2 \min \left\{-\alpha_{1} \alpha_{2} e^{i\left(\phi_{2}-\phi_{1}\right)}-\alpha_{1} \alpha_{3} e^{i\left(\phi_{3}-\phi_{1}\right)}-\alpha_{2} \alpha_{3} e^{i\left(\phi_{3}-\phi_{2}\right)}\right\} .
\end{aligned}
$$

The last equality above follows from (2.1). Thus,

$$
\begin{aligned}
b & =2 \max \left\{\alpha_{1} \alpha_{2}\left\langle u_{1}, u_{2}\right\rangle+\alpha_{1} \alpha_{3}\left\langle u_{1}, u_{3}\right\rangle+\alpha_{2} \alpha_{3}\left\langle u_{2}, u_{3}\right\rangle \mid\left(u_{1}, u_{3}, u_{3}\right) \in \mathcal{U}_{3}\right\} \\
& =2 \max \left\{\alpha_{1} \alpha_{2} e^{i\left(\phi_{2}-\phi_{1}\right)}+\alpha_{1} \alpha_{3} e^{i\left(\phi_{3}-\phi_{1}\right)}+\alpha_{2} \alpha_{3} e^{i\left(\phi_{3}-\phi_{2}\right)}\right\} .
\end{aligned}
$$

Analogously,

$$
\begin{aligned}
a & =2 \min \left\{\alpha_{1} \alpha_{2}\left\langle u_{1}, u_{2}\right\rangle+\alpha_{1} \alpha_{3}\left\langle u_{1}, u_{3}\right\rangle+\alpha_{2} \alpha_{3}\left\langle u_{2}, u_{3}\right\rangle \mid\left(u_{1}, u_{3}, u_{3}\right) \in \mathcal{U}_{3}\right\} \\
& =-2 \max \left\{-\alpha_{1} \alpha_{2}\left\langle u_{1}, u_{2}\right\rangle-\alpha_{1} \alpha_{3}\left\langle u_{1}, u_{3}\right\rangle-\alpha_{2} \alpha_{3}\left\langle u_{2}, u_{3}\right\rangle \mid\left(u_{1}, u_{3}, u_{3}\right) \in \mathcal{U}_{3}\right\} \\
& =-2 \max \left\{-\alpha_{1} \alpha_{2} e^{i\left(\phi_{2}-\phi_{1}\right)}-\alpha_{1} \alpha_{3} e^{i\left(\phi_{3}-\phi_{1}\right)}-\alpha_{2} \alpha_{3} e^{i\left(\phi_{3}-\phi_{2}\right)}\right\} \\
& =2 \min \left\{\alpha_{1} \alpha_{2} e^{i\left(\phi_{2}-\phi_{1}\right)}+\alpha_{1} \alpha_{3} e^{i\left(\phi_{3}-\phi_{1}\right)}+\alpha_{2} \alpha_{3} e^{i\left(\phi_{3}-\phi_{2}\right)}\right\} .
\end{aligned}
$$


Since the set

$$
Y_{\alpha_{1}, \alpha_{2}, \alpha_{3}}=\left\{2 \alpha_{1} \alpha_{2} e^{i\left(\phi_{2}-\phi_{1}\right)}+\alpha_{1} \alpha_{3} e^{i\left(\phi_{3}-\phi_{1}\right)}+\alpha_{2} \alpha_{3} e^{i\left(\phi_{3}-\phi_{2}\right)} \mid \phi_{1}, \phi_{2}, \phi_{3} \in \mathbb{R}\right\}
$$

is closed and convex, it is equal to $[a, b]$. Hence, $1-\alpha_{1}^{2}-\alpha_{2}^{2}-\alpha_{3}^{2} \in Y_{\alpha_{1}, \alpha_{2}, \alpha_{3}}$. It follows that there are complex numbers $e^{i \phi_{1}}, e^{i \phi_{2}}, e^{i \phi_{3}}$ which after substitution for $u, v, w$, correspondingly, in (3.2) give an identity. Thus, $z=\alpha_{1} e^{i \phi_{1}}+\alpha_{2} e^{i \phi_{2}}+\alpha_{3} e^{i \phi_{3}}$ has modulus one and $e^{i \phi_{1}}, e^{i \phi_{2}}, e^{i \phi_{3}}, z$ satisfy (3.1).

\section{REFERENCES}

1. S. Agnihotri, C. Woodward, Eigenvalues of products of unitary matrices and quantum Schubert calculus, Math. Research Letters 5 (1998), 817-836. MR.1671192 (2000a:14066)

2. M. Bakonyi, D. Timotin, Extensions of positive definite functions on free groups, J. Funct. Anal. 246 (2007), 31-49. MR2316876 (2008g:43009)

3. S. Corvez, F. Rouillier, Using computer algebra tools to classify serial manipulators, in Automated Deduction in Geometry. Lecture Notes in Comput. Sci., vol. 2930. Springer, 2004, pp. 31-43. MR2090401

4. K. Dykema, K. Juschenko, Matrices of unitary moments, arXiv:0901.0288.

5. W. Fulton, Eigenvalues, invariant factors, highest weights and Schubert calculus, Bull. Amer. Math. Soc. 37 (2000), 209-249. MR1754641 (2001g:15023)

6. K. Juschenko, S. Popovych, Algebraic reformulation of Connes' embedding problem and the free group algebra, Israel J. Math, in press.

7. E. Kirchberg, On nonsemisplit extensions, tensor products and exactness of group $C^{*}$ algebras, Invent. Math. 112 (1993), no. 3, 449-489. MR1218321 (94d:46058)

8. I. Klep, M. Schweighofer, Connes' embedding conjecture and sums of Hermitian squares, Adv. Math. 217 (2008), 1816-1837. MR2382741 (2009g:46109)

9. D. Lazard, F. Rouillier, Solving parametric polynomial systems, J. Symbolic Comput. 42 (2007), 636-667. MR2325919 (2008g:68131)

10. S. McCullough, Factorization of operator valid polynomials in several non-commuting variables, Linear Algebra Appl. 326 (2001), 193-203. MR1815959 (2002f:47035)

11. D. Mumford, The red book of varieties and schemes. In Lecture Notes in Mathematics, vol. 1358, Springer-Verlag, 1988. MR971985 (89k:14001)

12. S. Popovych, Positive semidefinite quadratic forms on unitary matrices, Linear Algebra Appl. 433 (2010), no. 1, 164-171.

13. S. Popovych, On $O^{*}$-representability and $C^{*}$-representability of $*$-algebras, Houston J. Math. 36 (2010), no. 2, 591-617.

14. A. Tarski, A Decision Method for Elementary Algebra and Geometry, University of California Press, 1951. MR0044472 (13:423a)

15. B. Wang, F. Zhang, A trace inequality for unitary matrices, Amer. Math. Monthly 101 (1994), no. 5, 453-455. MR1272947(95a:15015)

Department of Mathematical Sciences, Chalmers University of Technology, SE-412 96 GÖteborg, Sweden

E-mail address: popovych@univ.kiev.ua 